\title{
ZS Research Square \\ Fetal Sex-Dependent Associations Between Gestational Hormone Concentrations and Adverse Birth Outcomes
}

\section{Amber L. Cathey}

University of Michigan-Ann Arbor

Deborah J. Watkins

University of Michigan-Ann Arbor

Zaira Y. Rosario

University of Puerto Rico, Medical Sciences Campus

Carmen M. Vélez Vega

University of Puerto Rico, Medical Sciences Campus

Bhramar Mukherjee

University of Michigan-Ann Arbor

Marie S. O'Neill

University of Michigan-Ann Arbor

Rita Loch-Caruso

University of Michigan-Ann Arbor

Akram N. Alshawabkeh

Northeastern University

José F. Cordero

University of Georgia

John D. Meeker ( $\nabla$ meekerj@umich.edu )

University of Michigan-Ann Arbor

\section{Research Article}

Keywords: birth cohort, corticotropin releasing hormone, gestational age, preeclampsia, pregnancy, preterm birth, progesterone

Posted Date: January 26th, 2021

DOI: https://doi.org/10.21203/rs.3.rs-151002/v1

License: @ (i) This work is licensed under a Creative Commons Attribution 4.0 International License. Read Full License 


\section{Abstract}

Adverse birth outcomes remain significant public health problems that can have long-lasting impacts on mother and child. Understanding biological mechanisms underlying these outcomes, including altered endocrine function, can inform prevention efforts. The aim of this study was to evaluate associations between hormones at two times points during mid-gestation and adverse birth outcomes, and to assess effect modification by fetal sex. Repeated gestational hormone measurements (at 18 and 26 weeks) and birth outcomes were assessed among 976 women in PROTECT, a longitudinal prospective birth cohort in northern Puerto Rico, from 2011 to 2018. Birth outcomes assessed included preterm and spontaneous preterm birth (PTB), preeclampsia, gestational diabetes mellitus (GDM), small/large for gestational age (SGA, LGA), birthweight z-score, and gestational age at birth. Multivariate logistic and linear regressions were fit using visit-specific concentrations of hormones. We also conducted sensitivity analyses assessing impacts of fetal sex on observed associations. All models were adjusted for maternal age and education, and other confounders were assessed separately between birth outcomes based on a priori knowledge and observed associations with exposure and outcome measures. We observed increased odds of spontaneous PTB with IQR increases in progesterone (OR: 2.12, 95\% Cl: 1.29, 3.47), fT4 (OR: 1.73, 95\% Cl: 1.04, 2.86), and the ratio of progesterone to estriol (OR: 1.63, 95\% Cl: 1.05, 2.54$)$ at 26 weeks. Elevated estriol was protective against preeclampsia at 26 weeks (OR: $0.42,95 \% \mathrm{Cl}: 0.17,0.99)$. Increases in TSH and T3 conferred greater risk of GDM at 18 weeks. Many associations were modified by fetal sex, with hormone alterations during male pregnancies conferring greater risk of PTB, spontaneous PTB, and GDM. In conclusion, associations between hormones and birth outcomes vary based on timing of hormone measurement and fetal sex. Future studies are needed to understand mechanisms involved in adverse birth outcomes and fetal sex differences.

\section{Introduction}

Preterm birth (PTB) affects approximately $11 \%$ of live births ${ }^{1}$ and is the leading cause of neonatal mortality worldwide ${ }^{2}$. Infants born preterm are at increased risk for adverse health outcomes later in life including reduced renal function ${ }^{3}$, neurodevelopmental impairments ${ }^{4}$, cerebral palsy ${ }^{5}$, and reduced myocardial function ${ }^{6}$. Despite being a common public health problem, the causes of PTB are largely unknown. Other rare birth outcomes are also of significant concern and present safety issues for the mother and the fetus. The spontaneous subtype of preterm birth is characterized by an inflammatory uterine environment and may arise via different mechanisms than indicated $\mathrm{PTB}^{7}$. Preeclampsia, a hypertensive disorder of pregnancy ${ }^{8}$, affects $6 \%$ of pregnancies globally ${ }^{9}$ and is the leading cause of maternal mortality in the United States ${ }^{10,11}$. Gestational diabetes mellitus (GDM) is a disease of reduced insulin sensitivity and elevated glucose levels during gestation. High maternal glucose levels easily cross the placenta and illicit a response from the fetal pancreas. Infants born to mothers with GDM are at elevated risk for macrosomia and metabolic dysfunctions, and mothers become more likely to develop diabetes later in life ${ }^{12}$. Very little epidemiologic work has been done to investigate these rare adverse pregnancy outcomes and so our knowledge of the mechanisms by which they occur is limited.

The maternal and fetal endocrine milieus change and interact in unique ways at different points throughout gestation. The roles of progesterone are complex, reflected by the mixed efficacy of progesterone therapy as a preventative measure for preterm birth ${ }^{13}$. Estrogens are responsible for uterine maintenance and increased expression of oxytocin receptors and gap junctions that are necessary for uterine contractions to occur ${ }^{14}$. 
Through pregnancy, progesterone maintains uterine quiescence and keeps contractile effects of estrogens in check $^{13}$. Thyroid hormones are critical early in pregnancy for proper brain and skeletal development of the fetus $^{15}$. The maternal supply of thyroxine (T4) is particularly important in the first half of pregnancy, before the fetal thyroid gland has matured enough to produce adequate hormones ${ }^{16}$. Previous studies have demonstrated associations between clinical hyper- and hypothyroidism and adverse birth outcomes ${ }^{17-20}$, but much less is known about subclinical thyroid disruption and pregnancy outcomes.

Few large epidemiological studies exist that assess a wide array of hormone concentrations and pregnancy outcomes. The majority of existing research focuses on one hormone/class of hormones, which makes it challenging to gain a broad understanding of the endocrine pathways implicated in the onset of adverse birth outcomes $^{21-23}$. Specifically, the spontaneous subtype of PTB has not been well studied, and current research on the rare outcomes of preeclampsia and GDM is sparse ${ }^{24-26}$. Importantly, few previous studies have investigated hormone concentrations at more than one time point during gestation, nor have they assessed the impact of fetal sex on these associations. Because of these gaps in the literature, the aim of this study was to investigate associations between various hormone concentrations, measured at two time points during gestation, and adverse birth outcomes, as well as effect modification by fetal sex. Based on previous literature, we have hypothesized that increases in $\mathrm{CRH}$ and estriol will be associated with increased risk of early delivery, while increases in progesterone will be associated with later delivery. Further, we expect lower thyroid hormone concentrations to be associated with smaller infant size at birth. Finally, we expect to observe more significant adverse associations among male pregnancies, given previous evidence suggesting that male pregnancies are more risky than female pregnancies ${ }^{27-29}$.

\section{Methods}

\section{Study Population}

Pregnant women were recruited into the PROTECT birth cohort between 2011 and 2018 at $14 \pm 2$ weeks' gestation from seven hospitals and prenatal clinics in northern Puerto Rico. Study design and recruitment protocols have been described elsewhere ${ }^{30}$. Demographic and self-reported health information was provided at the first clinic visit. This study was approved by the research and ethics committees of the University of Michigan School of Public Health, University of Puerto Rico, Northeastern University, and participating hospitals and clinics. All methods reported in this study were performed in accordance with relevant guidelines and regulations imposed by those institutions. All study participants provided full informed consent prior to participation.

\section{Hormone Measurements}

All women provided serum samples at their first and third clinic visits, aligning with median 18 (range 16-20) and 26 (range 24-28) weeks' gestation. Serum samples were analyzed at the Central Ligand Assay Satellite Services (CLASS) laboratory in the Department of Epidemiology at the University of Michigan School of Public Health. Progesterone, sex hormone-binding globulin (SHBG), testosterone, total triiodothyronine (T3), total thyroxine (T4), free thyroxine (fT4), and thyroid-stimulating hormone (TSH) were measured using a chemiluminescence immunoassay. Estriol and corticotropin releasing hormone $(\mathrm{CRH})$ were measured using an enzyme immunoassay. Some hormone concentrations were not available for all participants due to sample volume limitations. The ratios of progesterone to estriol (Prog/E3) and T3 to T4 (T3/T4) were assessed in 
addition to measured hormones because of previous research indicating that the ratios may be better indices of adverse pregnancy outcomes than single hormone measurements $21,31,32$. All hormone concentrations below the limit of detection (LOD) were replaced by the LOD divided by the square root of two.

\section{Birth Outcome Assessment}

Based on recommendations from the American College of Obstetricians and Gynecologists, self-reported date of the last menstrual period was collected at the first study visit and used in combination with early ultrasound measurements to determine gestational age at birth ${ }^{33}$. PTB was defined as delivery before 37 weeks' gestation. We also assessed spontaneous PTB, defined as PTB presenting with premature rupture of membranes, spontaneous preterm labor, or both ${ }^{7}$. Preeclampsia and GDM cases were determined based on diagnosis in the medical record by an attending physician. We calculated birthweight z-scores based on fetal sex and gestational age using widely accepted international standards ${ }^{34}$. Those born with a birthweight $<10$ th percentile and $>90$ th percentile were considered small and large for gestational age (SGA and LGA), respectively.

\section{Statistical Methods}

Distributions of demographic, health, and pregnancy characteristics were calculated. Summary measures of gestational hormone concentrations were assessed using arithmetic means of all available concentrations for each study participant, or geometric means for log-normally distributed hormones. Distributions of hormone concentrations were also assessed individually at each study visit. Univariate linear models were used to test for significant differences between hormone concentrations at each study visit. Intraclass correlation coefficients (ICCS) were also used to assess between- and within-individual variability of hormone concentrations across study visits.

We utilized indicator variables for study visit and included interaction terms between each indicator and hormone concentration in final models to achieve effect estimates specific to each study visit. Sandwich estimators were used in these models to correct for biased standard errors due to the non-repeating nature of outcome variables. Gestational average hormone concentrations were not used in final statistical models because of the marked changes in some hormones that occur throughout gestation. We also conducted sensitivity analyses to assess effect modification by fetal sex. An additional interaction term was included between hormone concentration and a fetal sex indicator variable to achieve effect estimates specific to fetal sex within study visits.

Confounders were explored by evaluating their associations with exposure and outcome variables. All models adjusted for categorical forms of maternal age and maternal education. Further covariate adjustment differed between birth outcomes based on a priori knowledge, significant association with the outcome measure, and inclusion of the covariate impacting the hormone effect estimate by at least $10 \%$. A list of covariates that were assessed and the outcome models in which they were included, if any, is shown in Table 1. All models assessing testosterone also included SHBG to adjust for bound testosterone. 
Table 1

Inclusion of covariates between different outcome models.

\begin{tabular}{|c|c|c|c|c|c|c|c|c|}
\hline & PTB & $\begin{array}{l}\text { Spontaneous } \\
\text { PTB }\end{array}$ & $\begin{array}{l}\text { Gestational } \\
\text { Age }\end{array}$ & $\begin{array}{l}\text { Birthweight } \\
\text { Z-Score }\end{array}$ & SGA & LGA & Preeclampsia & GDM \\
\hline Maternal Age & $x$ & $x$ & $x$ & $x$ & $x$ & $x$ & $x$ & $x$ \\
\hline $\begin{array}{l}\text { Maternal } \\
\text { Education }\end{array}$ & $x$ & $x$ & $x$ & $x$ & $\mathrm{x}$ & $x$ & $x$ & $x$ \\
\hline \multicolumn{9}{|l|}{$\begin{array}{l}\text { Employment } \\
\text { Status }\end{array}$} \\
\hline \multicolumn{9}{|l|}{$\begin{array}{l}\text { Annual } \\
\text { Household } \\
\text { Income }\end{array}$} \\
\hline Marital Status & $x$ & $x$ & $x$ & & & & & \\
\hline $\begin{array}{l}\text { Smoking } \\
\text { Status }\end{array}$ & & & & & $x$ & $x$ & & \\
\hline $\begin{array}{l}\text { Environmental } \\
\text { Tobacco } \\
\text { Smoke } \\
\text { Exposure }\end{array}$ & $x$ & $\mathrm{x}$ & $x$ & & & & & $x$ \\
\hline Alcohol Usage & & & $x$ & & & & & $x$ \\
\hline \multicolumn{9}{|l|}{ Parity } \\
\hline $\begin{array}{l}\text { Pre-Pregnancy } \\
\text { BMI }\end{array}$ & & & $x$ & $x$ & & & $x$ & \\
\hline Infant Sex & & & & & & & & \\
\hline
\end{tabular}

\section{Results}

Demographics of the study population are shown in Table 2 . The majority of mothers were under the age of 30 (67.1\%), had at least some college education (79\%), were employed (63\%), had an annual household income under $\$ 30,000(63.1 \%)$, were married $(53.1 \%)$, had never smoked $(86 \%)$ or been exposed to environmental tobacco smoke (88.7\%), did not drink alcohol during pregnancy (93.6\%), had given birth to less than 2 previous children (86.9\%), and had a pre-pregnancy BMI of less than $25(56.1 \%)$. 
Table 2

Maternal demographic characteristics of the study population ( $\mathrm{N}$ =976)

\begin{tabular}{|c|c|}
\hline & $\mathbf{N}(\%)$ \\
\hline \multicolumn{2}{|l|}{ Maternal Age (years) } \\
\hline $18-24$ & $354(36.3 \%)$ \\
\hline $25-29$ & $301(30.8 \%)$ \\
\hline $30-34$ & $206(21.1 \%)$ \\
\hline $35-41$ & $115(11.8 \%)$ \\
\hline \multicolumn{2}{|l|}{ Maternal Education } \\
\hline GED or less & $203(21 \%)$ \\
\hline Some College & $331(34.2 \%)$ \\
\hline Bachelors or Higher & $433(44.8 \%)$ \\
\hline \multicolumn{2}{|l|}{ Employment Status } \\
\hline No & $357(37 \%)$ \\
\hline Yes & $608(63 \%)$ \\
\hline \multicolumn{2}{|c|}{ Annual Household Income } \\
\hline$<10 \mathrm{k}$ & $269(31.6 \%)$ \\
\hline $10 k-<30 k$ & $268(31.5 \%)$ \\
\hline $30 \mathrm{k}-<50 \mathrm{k}$ & $203(23.8 \%)$ \\
\hline$>=50 \mathrm{k}$ & $112(13.1 \%)$ \\
\hline \multicolumn{2}{|l|}{ Marital Status } \\
\hline Single & $197(20.4 \%)$ \\
\hline Married & $521(53.9 \%)$ \\
\hline Cohabitating & $249(25.7 \%)$ \\
\hline \multicolumn{2}{|l|}{ Smoking Status } \\
\hline Never & $833(86 \%)$ \\
\hline Ever & $121(12.5 \%)$ \\
\hline Current & $15(1.55 \%)$ \\
\hline \multicolumn{2}{|c|}{ Daily Environmental Tobacco Smoke Exposure } \\
\hline Never & $808(88.7 \%)$ \\
\hline 1 Hour or less & $40(4.39 \%)$ \\
\hline
\end{tabular}




\begin{tabular}{|ll|}
\hline & N (\%) \\
\hline Alcohol Use & $63(6.92 \%)$ \\
\hline Never & \\
\hline Yes, before Pregnancy & $504(52.2 \%)$ \\
\hline Yes, currently & $400(41.4 \%)$ \\
\hline Number of Previous Children & $62(6.42 \%)$ \\
\hline 0 & \\
\hline 1 & $355(42.7 \%)$ \\
\hline 2 to 5 & $367(44.2 \%)$ \\
\hline Pre-Pregnancy BMl & $109(13.1 \%)$ \\
\hline$[0,25]$ & \\
\hline$(25,30]$ & $520(56.1 \%)$ \\
\hline Above 30 & $240(25.9 \%)$ \\
\hline Fetal Sex & $167(18 \%)$ \\
\hline Female & \\
\hline Male & $464(48 \%)$ \\
\hline
\end{tabular}

Distributions of hormone concentrations are shown in Supplementary Table S1. Most hormone concentrations were significantly different at 18 and 26 weeks' gestation, with notable increases occurring with estriol (median 15.1 and $38.2 \mathrm{ng} / \mathrm{mL}$ at 18 and 26 weeks, respectively) and progesterone (median 39.3 and $73.5 \mathrm{ng} / \mathrm{mL}$ at 18 and 26 weeks, respectively). ICCs for all other hormones ranged from 0.647 (T4) to 0.856 (testosterone).

Distributions of birth outcomes are shown in Table 3. PTB and spontaneous PTB occurred in $9.9 \%$ and $5.8 \%$ of the study population, respectively. Preeclampsia and GDM were less prevalent ( $2.9 \%$ and $1.9 \%$, respectively). Occurrences of SGA and LGA births were similar ( $8.9 \%$ and $9.6 \%$, respectively). Median gestational age of the study population was 39.1 weeks (IQR: 38.1-40). 
Table 3

Distributions of continuous and binary birth outcomes

\begin{tabular}{|c|c|c|c|c|c|c|c|}
\hline & Min & 10th & 25th & 50th & 75th & 90th & Max \\
\hline Gestational Age (wks) & 20.3 & 36.7 & 38.1 & 39.1 & 40 & 40.7 & 42.7 \\
\hline \multirow[t]{3}{*}{ Birthweight Z-Score (ounces) } & -5.34 & -1.19 & -0.571 & -0.00005 & 0.707 & 1.25 & 9.70 \\
\hline & $(19.0)$ & $(91.0)$ & $(102)$ & $(113)$ & (123) & (133) & (224) \\
\hline & \multicolumn{7}{|l|}{$N(\%)$} \\
\hline \multicolumn{8}{|l|}{ Preterm Birth } \\
\hline No & \multicolumn{7}{|c|}{$867(90.1 \%)$} \\
\hline Yes & \multicolumn{7}{|c|}{$95(9.88 \%)$} \\
\hline \multicolumn{8}{|l|}{ Spontaneous Preterm Birth } \\
\hline No & \multicolumn{7}{|c|}{$883(94.2 \%)$} \\
\hline Yes & \multicolumn{7}{|c|}{$54(5.76 \%)$} \\
\hline \multicolumn{8}{|l|}{ Preeclampsia } \\
\hline No & \multicolumn{7}{|c|}{$947(97.1 \%)$} \\
\hline Yes & \multicolumn{7}{|c|}{$28(2.87 \%)$} \\
\hline \multicolumn{8}{|l|}{ Gestational Diabetes } \\
\hline No & \multicolumn{7}{|c|}{$900(98.1 \%)$} \\
\hline Yes & \multicolumn{7}{|c|}{$17(1.85 \%)$} \\
\hline \multicolumn{8}{|l|}{ Small for Gestational Age } \\
\hline No & \multicolumn{7}{|c|}{$842(91.1 \%)$} \\
\hline Yes & \multicolumn{7}{|c|}{$82(8.87 \%)$} \\
\hline \multicolumn{8}{|l|}{ Large for Gestational Age } \\
\hline No & \multicolumn{7}{|c|}{$835(90.4 \%)$} \\
\hline Yes & \multicolumn{7}{|c|}{89 (9.63\%) } \\
\hline
\end{tabular}

Figure 1 shows the associations between hormone concentrations and birth outcomes at each study visit (all effect estimates and p-values are shown in Supplementary Table S2). There were greater odds of spontaneous PTB with increasing progesterone concentrations at 26 weeks (OR: 2.12, 95\% Cl: 1.29, 3.47) and fT4 concentrations at both study visits (18wk OR: 1.60, 95\% Cl: 1.07, 2.39; 26wk OR: 1.73, 95\% Cl: 1.04, 2.86). The risk of spontaneous PTB was significantly different between study visits with an IQR increase in Prog/E3 (interaction $p=0.026$ ), a null association observed at 18 weeks and increased odds observed at 26 weeks (OR: $1.63,95 \% \mathrm{Cl}$ : $1.05,2.54)$. Reductions in gestational age at birth were observed with increased concentrations of progesterone ( $\beta$ : -3.56 days, $95 \%$ Cl: $-6.02,-1.10)$, fT4 ( $\beta$ : -2.22 days, $95 \%$ Cl: $-3.84,-0.61$ ), and T4 ( $\beta:-1.87$ days, $95 \%$ Cl: -3.62 , -0.11 ) around 18 weeks, and with prog/e3 at both study visits (18wk $\beta$ : -1.77 days, $95 \% \mathrm{Cl}:-3.36,-0.19 ; 26 \mathrm{wk} \beta$ : 
-1.98 days, $95 \% \mathrm{Cl}:-3.58,-0.37)$. Notably, the effect of progesterone was significantly different between study visits (interaction $\mathrm{p}=0.044$ ).

Results at 18 weeks suggested that elevated progesterone and reduced estriol are associated with increased risk of having an SGA infant (E3 OR: 0.66, 95\% Cl: 0.45, 0.97; progesterone OR: 1.53, 95\% Cl: 1.09, 2.17; prog/E3 OR: 1.77, 95\% Cl: 1.29, 2.44). This trend remained at 26 weeks for only prog/E3 (OR: 1.53, 95\% Cl: 1.07, 2.17). Similarly, prog/E3 at 18 weeks was inversely associated with birthweight z-score $(\beta:-0.12,95 \% \mathrm{Cl}$ : $-0.23,-0.02)$ and estriol at 26 weeks was positively associated with birthweight z-score ( $\beta$ : $0.21,95 \% \mathrm{Cl}: 0.01,0.41)$.

A protective effect against preeclampsia was observed with increases in SHBG at 18 weeks (OR: 0.55, 95\% Cl: $0.30,0.99)$ and estriol (OR: 0.42, 95\% Cl: 0.17, 0.99) and SHBG (OR: 0.46, 95\% Cl: 0.25, 0.83) at 26 weeks. Conversely, elevated risk of preeclampsia was observed with an increase in TSH at 26 weeks (OR: 2.18, 95\% $\mathrm{Cl}$ : 1.19, 3.99). The odds of GDM increased with an IQR increase in TSH (OR: 1.67, 95\% Cl: 1.02, 2.72), T3 (OR: 2.83 , 95\% Cl: 1.04, 7.68), and T3/T4 (OR: 2.97, 95\% Cl: 1.20, 7.35) at 18 weeks, and increased with higher estriol at 18 weeks (OR: $5.95,95 \% \mathrm{Cl}: 1.27,27.8)$. None of the associations with preeclampsia or GDM were significantly different between study visits.

Sensitivity analyses revealed that many associations were significantly different between male and female pregnancies (Fig. 2; all effect estimates and p-values are shown in Supplementary Table S3). The most compelling effect modification by fetal sex was observed for preterm birth; the interaction term between hormone concentration and fetal sex indicator was significant among 7 out of 11 hormones and hormone ratios assessed. SHBG was protective against PTB at 26 weeks among female (OR: 0.60, 95\% Cl: 0.37, 0.96), but not male, pregnancies (interaction $\mathrm{p}=0.032$ ). Higher testosterone at both study visits was associated with increased odds of PTB among female pregnancies and reduced odds of PTB among male pregnancies (interaction $\mathrm{p}<0.001$ ). Notably, increased odds of PTB were observed among only male pregnancies with elevated concentrations of CRH (OR: 1.82, 95\% Cl: 1.09, 3.05; interaction p = 0.002), estriol (OR: 1.81, 95\% Cl: 1.07, 3.06; interaction $p=$ 0.022), progesterone (OR: 1.88, 95\% Cl: 1.16, 3.04; interaction $\mathrm{p}=0.011)$, and fT4 (OR: 1.63, 95\% Cl: 1.06, 2.51; interaction $p=0.115$ ) at 18 weeks. Assessment of gestational age as a continuous variable did not provide such compelling results, but it did provide additional evidence of fetal sex modifying the association with progesterone at 18 weeks (male pregnancy $\beta$ : -4.9 days, $95 \% \mathrm{Cl}:-2.73,-7.07$ days; interaction $\mathrm{p}=0.015$ ).

The spontaneous subtype of PTB also showed several cases of effect modification by fetal sex. An IQR increase in $\mathrm{CRH}$ at 18 weeks was associated with greater odds of spontaneous PTB among only male pregnancies (OR: $2.73,95 \% \mathrm{Cl}$ : 1.38, 5.43; interaction $\mathrm{p}=0.003)$. Increases in testosterone at both visits were protective against spontaneous PTB among only male pregnancies (interaction $p=0.001$ ). Increases in T3 and fT4 at both study visits were associated with increased odds of spontaneous PTB among only male pregnancies, but effect modification was significant only for T3 (interaction $p=0.013$ ). Finally, higher progesterone at 26 weeks was associated with increased off of spontaneous PTB among only male pregnancies (OR: 2.34, 95\% Cl: 1.36, 4.03).

Fetal sex modified the association between SGA and only the ratio prog/E3 (interaction $\mathrm{p}=0.022$ ), which was positive among only male pregnancies at both 18 weeks (OR: 2.39, 95\% Cl: 1.59, 3.60) and 26 weeks (OR: 1.98 , $95 \% \mathrm{Cl}: 1.29,3.05)$. Accordingly, increased estriol resulted in increases in birthweight z-score at both 18 weeks ( $\beta$ : $0.19,95 \% \mathrm{Cl}: 0.02,0.36)$ and 26 weeks $(\beta: 0.31,95 \% \mathrm{Cl}: 0.08,0.53)$ among only male pregnancies (interaction $\mathrm{p}=$ 0.030). Fetal sex did not modify any associations between hormones and odds of LGA. 
Though there was no evidence of effect modification by fetal sex on associations between hormones and preeclampsia, significant effects were observed only among female pregnancies with increases in SHBG (OR: $0.34,95 \% \mathrm{Cl}: 0.14,0.81$ ), TSH (OR: $2.41,95 \% \mathrm{Cl}: 1.11,5.23$ ), and fT4 (OR: $0.40,95 \% \mathrm{Cl}: 0.17,0.92$ ) at 26 weeks. Conversely, there was significant evidence of effect modification by fetal sex on the association between various hormones and odds of GDM. Elevated thyroid hormones were observed to be protective against GDM among female pregnancies [(fT4 at 18wks OR: $0.29,95 \% \mathrm{Cl}: 0.10,0.85$; interaction $p=0.001)$, (T4 at 18wks OR: $0.32,95 \%$ Cl: $0.11,0.90$; interaction $p=0.002)$ ], but positively associated with GDM among male pregnancies [(T3 at $18 \mathrm{wks}$ OR: $6.04,95 \% \mathrm{Cl}: 1.72,21.3$; interaction $\mathrm{p}=0.028$ ), (fT4 at $26 \mathrm{wks}$ OR: $4.87,95 \% \mathrm{Cl}: 1.53,15.5)$, (T4 at $26 \mathrm{wks}$ OR: $3.05,95 \% \mathrm{Cl}: 1.02,9.13)]$. A similar trend was observed for the ratio of prog/E3; there was a protective effect at 18 weeks among female pregnancies (OR: $0.25,95 \% \mathrm{Cl}: 0.09,0.71)$ and a positive association at 26 weeks among male pregnancies (OR: $2.93,95 \% \mathrm{Cl}: 0.99,8.69$; interaction $\mathrm{p}=0.004)$.

\section{PTB and Gestational Age}

We observed greater odds of PTB and spontaneous PTB with increasing progesterone concentrations (when fetal sex was male), but other studies demonstrating similar significant associations are lacking. One study observed progesterone concentrations measured between 28 and 32 weeks' gestation to be higher among women who delivered preterm compared to full term ${ }^{22}$. We observed higher progesterone concentrations among PTB cases when fetal sex was male, but only around 18 weeks' gestation. We also observed higher progesterone concentrations around 26 weeks among women who spontaneously delivered preterm compared to women who carried to term.

Previous work has shown that a ratio favoring estriol in mid-pregnancy ${ }^{21}$ and at delivery ${ }^{35}$ is associated with earlier time of labor. Progesterone concentrations rise steadily during pregnancy, contributing to uterine quiescence, downregulation of prostaglandin production, and immune tolerance of the fetus ${ }^{36,37}$. At the onset of human labor, progesterone concentrations do not notably decrease; rather, the body's response to progesterone is dampened. It is not clear exactly how this occurs, but possibilities include reduction in progesterone receptor expression, changes in receptor isoforms, and local progesterone metabolism ${ }^{38}$. As term approaches, the ratio of progesterone to estriol shifts to favor estrogens, with the functional decrease in progesterone driving initiation of labor $^{39}$. The new dominance of estrogens promotes an increase in prostaglandin and oxytocin receptors and enzymes responsible for muscle contractions, which work together to help promote labor ${ }^{40}$. We observed a positive association between odds of PTB and estriol concentrations (when fetal sex was male), but we also unexpectedly observed later gestational age at birth with higher concentrations of estriol at 26 weeks' gestation when the fetus was female. In contrast with previous studies, we observed that higher prog/E3 was associated with reduced gestational age and increased odds of SGA. Interestingly, among women who delivered preterm, a previous study observed lower prog/E3 among only those without premature rupture of membranes ${ }^{41}$, possibly implicating different endocrine pathways in the occurrence of PTB with and without premature rupture of membranes.

Decreased odds of PTB have been shown with increased concentrations of fT4 in the second ${ }^{23}$ and third 42 trimesters, which contradicts our finding that fT4 was inversely associated with gestational age at birth (among the whole study population and when the fetus was male), and increased odds of PTB (when the fetus was male) and spontaneous PTB. One prior study also found increased odds of PTB with greater T3 concentrations at 10 
and 26 weeks gestation ${ }^{42}$. Similarly, we found that T3 was associated with spontaneous PTB when the fetus was male. Mechanisms of the association between thyroid hormones and PTB are poorly understood, but previous research has suggested that altered thyroid hormone concentrations may be involved in other disease states or exposures for which we have evidence of associations with PTB such as oxidative stress and inflammation ${ }^{43-45}$, or environmental exposures such as phthalates ${ }^{46-48}$.

Several previous studies have observed that male fetal sex is associated with a greater risk of delivering preterm. Proposed biological explanations for this observation include a pro-inflammatory environment generated by a male fetus ${ }^{27}$ and larger size at birth for males relative to females ${ }^{28}$. Increased risk of PTB when the fetus was male among only Caucasian women has also been observed, suggesting a potential interaction between race and fetal sex ${ }^{29}$. We observed significant associations with PTB unique to women carrying a male fetus for $\mathrm{CRH}$, estriol, progesterone, and fT4, providing further evidence that the effect of fetal sex on the occurrence of PTB is complex, possibly involving diverse endocrine pathways.

\section{Preeclampsia}

Among all pregnancies, we observed reduced odds of preeclampsia with an increase in estriol at 26 weeks. In accordance with our findings, another study showed that estriol concentrations in the second trimester ${ }^{49}$ were lower among women with preeclampsia than women with normal pregnancies. Previous studies have also found increased odds of preeclampsia with higher second trimester fT4 concentrations ${ }^{23,50}$, and lower third trimester fT4 concentrations ${ }^{51}$. All associations we observed between fT4 and preeclampsia were inverse, and the inverse association at 26 weeks among female pregnancies was significant. The association between fT4 and preeclampsia has been shown to be modified by human chorionic gonadotropin (hCG) concentrations, with high fT4 positively associated with preeclampsia only when hCG is low ${ }^{52}$. This effect modification may be due to the known angiogenic role of hCG during early pregnancy ${ }^{53}$.

Hormonal involvement in the etiology of preeclampsia is complex due to the angiogenic dysfunction of the affected uterus. In preeclampsia cases, proper remodeling and infiltration of blood vessels by placental extravillous trophoblasts does not occur, and this can be observed before the onset of clinical symptoms ${ }^{54,55}$. It is unclear whether endocrine disruption plays a causal role in initiation of uterine dysfunction, or if uterine dysfunction triggers a maternal endocrine response in an attempt to adapt to the hypoxic state ${ }^{56}$.

\section{Gestational Diabetes Mellitus}

A previous epidemiology study has demonstrated associations between high second trimester estriol concentrations and greater odds of $\mathrm{GDM}^{25}$. We also observed increased odds of GDM with estriol at 26 weeks. Testosterone concentrations were inversely associated with odds of GDM among male pregnancies in our study, which differs from previous research that showed higher testosterone concentrations among women with GDM ${ }^{26}$ and with greater insulin resistance ${ }^{57}$ compared to women with normal pregnancies.

Previous work has suggested that fT4 concentrations early in pregnancy are inversely associated with odds of $\mathrm{GDM}^{51,58}$. In accordance with those findings, the ratio of fT3 to fT4 has been observed to be positively associated with odds of $\mathrm{GDM}^{59}$, suggesting that increased conversion of T4 to biologically active T3 may play a role in the onset of GDM. In alignment with those findings, we observed greater odds of GDM among all pregnancies with increased T3 concentrations at 18 weeks, and greater odds of GDM among male pregnancies 
with increased T3 at both study visits. We also observed an inverse association between fT4 and odds of GDM at

18 weeks among female pregnancies, while that association was positive among male pregnancies at 26 weeks. Previous work has shown that women with GDM have higher circulating concentrations of inflammatory cytokines such as IL-6 and TNF-alpha ${ }^{60}$, which have been observed to be inversely associated with T3 concentrations ${ }^{61}$. These inflammatory markers may increase insulin resistance during pregnancy and, mediated by alterations in thyroid hormone concentrations, contribute to higher circulating glucose levels and increased odds of GDM. Several previous studies have observed greater risks for GDM among women carrying a male fetus $^{62-64}$, possibly due to poorer beta-cell function among male fetuses ${ }^{65}$.

\section{Birth Size}

We observed that decreased birthweight among females was marginally associated with elevated T4 at 26 weeks. Previous work found similar inverse associations, but with fT4 instead of total T4 ${ }^{66,67}$. Thyroid hormones are critical for fetal growth, possibly via their influences on fetal insulin-like growth factor, leptin, or the placenta's abilities to transfer nutrients ${ }^{68}$. Even in the case of nearly identical patterns of thyroid hormone concentrations throughout gestation between mothers, differences in expression of hormone transporters in the placenta and intracellular receptors in fetal tissues can result in different thyroid hormone exposure profiles for the fetus and, consequently, varying effects on fetal growth and development ${ }^{69}$. Assessment of thyroid hormone effects on birth outcomes in the second half of gestation is even more complex as the fetal thyroid gland begins to produce hormones and the fetus relies less on maternal supply of $\mathrm{T} 4{ }^{69}$. Conflicting results on the relationship between thyroid hormones and birthweight between studies may be due in part to unmeasured differences in fetal thyroid function.

\section{Strengths and Limitations}

The present study was subject to several limitations. We were not able to measure hCG or assess thyroid autoantibody status. Thus some of our results could be biased due to unmeasured confounding variables. Some critical changes in the maternal endocrine environment occur later in gestation than we were able to measure, such as the exponential increase in $\mathrm{CRH}$ right before the onset of labor. Although the goal of this study was to determine whether mid-pregnancy hormone levels were indicative of increased risk of adverse pregnancy outcomes, measurements at later time points could shed additional light on the various endocrine pathways implicated in adverse birth outcomes. We observed low rates of preeclampsia and GDM, which reduces the reliability of effect estimates. However, these lower rates were observed because we excluded women with preexisting conditions from our cohort to allow more precise examination of associations between hormone concentrations and birth outcomes, since preexisting conditions can influence hormone concentrations and susceptibility to adverse birth outcomes. Furthermore, excluding women with preexisting conditions may limit the

generalizability of our findings. Finally, some results assessing preeclampsia and GDM may be subject to reverse causation bias if the disease state, before clinical observation, resulted in the hormonal changes that we observed.

Despite the aforementioned limitations, this study was also strong in various ways. This is one of few studies to assess a broad panel of hormone concentrations at more than one time point during gestation to investigate relationships with various birth outcomes and different windows of susceptibility. Many epidemiological studies limit their analytical panel to either thyroid or steroid hormones, or do not assess the spontaneous subtype of PTB. We are also one of few groups to assess interactions between gestational hormone concentrations and 
fetal sex. Finally, our study was strengthened by a higher sample size of mothers than was seen in most previously published cohorts, which is particularly important when studying rare outcomes occurring in less than $5 \%$ of the population.

\section{Conclusions}

In conclusion, we observed a range of associations between hormones and adverse birth outcomes. We found differences based on the timing of hormone assessment, and many significant findings were unique to mothers carrying a male fetus. Future work will attempt to place these findings in the context of relevant environmental contaminants on the island of Puerto Rico by exploring possibilities of endocrine disruption as a mediator between chemical exposures and pregnancy outcomes. Additional studies are needed to more fully elucidate the role of altered hormone concentrations in the etiology of adverse birth outcomes.

\section{Declarations}

\section{Author Contributions}

ALC analyzed and interpreted data and drafted the original article. JDM, JFC, ANA, RLC, and CMV made substantial contributions to the conception and design of the study. DJW and ZYR contributed to acquisition of data. BM, RLC, and MSO assisted with interpretation of data. All authors provided substantial revisions and gave approval for the final version to be published.

\section{Acknowledgements}

We would like to extend our gratitude to all PROTECT study participants and their families. The authors also thank the nurses and research staff who participated in cohort recruitment and follow up, as well as the Federally Qualified Health Centers (FQHC) in Puerto Rico that facilitated participant recruitment, including Morovis Community Health Center, Prymed in Ciales, Camuy Health Services, Inc. and the Delta OBGyn Group in Manati, as well as the Manati Medical Center and the Metro Pavia Hospital in Arecibo.

\section{Additional Information}

This study was supported by the Superfund Research Program of the National Institute of Environmental Health Sciences (NIEHS), National Institutes of Health (NIH; grant number P42ES017198). Additional support was provided from NIEHS grant number P30ES017885 and the Environmental influences on Child Health Outcomes (ECHO) program grant number UH3OD023251. ECHO is a nationwide research program supported by the $\mathrm{NIH}$, Office of the Director to enhance child health.

\section{References}

1. Vogel, J. P. et al. The global epidemiology of preterm birth. Best Pract. Res. Clin. Obstet. Gynaecol.52, 3-12 (2018).

2. Blencowe, H. et al. National, regional, and worldwide estimates of preterm birth rates in the year 2010 with time trends since 1990 for selected countries: a systematic analysis and implications. Lancet (London, England).379, 2162-2172 (2012). 
3. Luyckx, V. A. Preterm Birth and its Impact on Renal Health. Semin. Nephrol.37, 311-319 (2017).

4. Leung, M. P., Thompson, B., Black, J., Dai, S. \& Alsweiler, J. M. The effects of preterm birth on visual development. Clin. Exp. Optom.101, 4-12 (2018).

5. Spittle, A. J., Morgan, C., Olsen, J. E., Novak, I. \& Cheong, J. L. Y. Early Diagnosis and Treatment of Cerebral Palsy in Children with a History of Preterm Birth. Clin. Perinatol.45, 409-420 (2018).

6. Schubert, U., Muller, M., Abdul-Khaliq, H. \& Norman, M. Preterm Birth Is Associated with Altered Myocardial Function in Infancy. J. Am. Soc. Echocardiogr.29, 670-678 (2016).

7. McElrath, T. F. et al. Pregnancy disorders that lead to delivery before the 28th week of gestation: an epidemiologic approach to classification. Am. J. Epidemiol.168, 980-989 (2008).

8. Hypertension in pregnancy. Report of the American College of Obstetricians and Gynecologists' Task Force on Hypertension in Pregnancy. Obstet. Gynecol.122, 1122-1131 (2013).

9. Hogan, M. C. et al. Maternal mortality for 181 countries, 1980-2008: a systematic analysis of progress towards Millennium Development Goal 5. Lancet (London, England).375, 1609-1623 (2010).

10. Kuklina, E. V., Ayala, C. \& Callaghan, W. M. Hypertensive disorders and severe obstetric morbidity in the United States. Obstet. Gynecol.113, 1299-1306 (2009).

11. Wanderer, J. P. et al. Epidemiology of obstetric-related ICU admissions in Maryland: 1999-2008*. Crit. Care Med.41, 1844-1852 (2013).

12. Coustan, D. R. Gestational diabetes mellitus. Clin. Chem.59, 1310-1321 (2013).

13. Sykes, L. \& Bennett, P. R. Efficacy of progesterone for prevention of preterm birth. Best Pract. Res. Clin. Obstet. Gynaecol.52, 126-136 (2018).

14. Weiss, G. Endocrinology of parturition. J. Clin. Endocrinol. Metab.85, 4421-4425 (2000).

15. Yen, P. M. Physiological and molecular basis of thyroid hormone action. Physiol. Rev.81, 1097-1142 (2001).

16. Springer, D., Jiskra, J., Limanova, Z., Zima, T. \& Potlukova, E. Thyroid in pregnancy: From physiology to screening. Crit. Rev. Clin. Lab. Sci.54, 102-116 (2017).

17. Mannisto, T. et al. Thyroid diseases and adverse pregnancy outcomes in a contemporary US cohort. J. Clin. Endocrinol. Metab.98, 2725-2733 (2013).

18. Allan, W. C. et al. Maternal thyroid deficiency and pregnancy complications: implications for population screening. J. Med. Screen.7, 127-130 (2000).

19. Abalovich, M. et al. Overt and subclinical hypothyroidism complicating pregnancy. Thyroid.12, 63-68 (2002).

20. Nazarpour, S., Ramezani Tehrani, F., Simbar, M. \& Azizi, F. Thyroid dysfunction and pregnancy outcomes. Iran. J. Reprod. Med.13, 387-396 (2015).

21. Ruiz, R. J. et al. The effect of acculturation on progesterone/estriol ratios and preterm birth in hispanics. Obstet. Gynecol.111, 309-316 (2008).

22. Feng, T. et al. The association between serum progesterone level and preterm delivery. Int. J. Gynaecol. Obstet.142, 308-314 (2018).

23. Korevaar, T. I. M. et al. Maternal total T4 during the first half of pregnancy: physiologic aspects and the risk of adverse outcomes in comparison with free T4. Clin. Endocrinol. (Oxf).85, 757-763 (2016).

24. Shin, Y. Y. et al. Regulation of steroid hormones in the placenta and serum of women with preeclampsia. Mol. Med. Rep.17, 2681-2688 (2018). 
25. Hur, J., Cho, E. H., Baek, K. H. \& Lee, K. J. Prediction of Gestational Diabetes Mellitus by Unconjugated Estriol Levels in Maternal Serum. Int. J. Med. Sci.14, 123-127 (2017).

26. Gozukara, Y. M. et al. Role of first trimester total testosterone in prediction of subsequent gestational diabetes mellitus. J. Obstet. Gynaecol. Res.41, 193-198 (2015).

27. Challis, J., Newnham, J., Petraglia, F., Yeganegi, M. \& Bocking, A. Fetal sex and preterm birth. Placenta.34, 95-99 (2013).

28. McGregor, J. A., Leff, M., Orleans, M. \& Baron, A. Fetal gender differences in preterm birth: findings in a North American cohort. Am. J. Perinatol.9, 43-48 (1992).

29. Wilms, F. F. et al. The impact of fetal gender and ethnicity on the risk of spontaneous preterm delivery in women with symptoms of preterm labor. J. Matern. Fetal. Neonatal Med.29, 3563-3569 (2016).

30. Meeker, J. D. et al. Distribution, variability, and predictors of urinary concentrations of phenols and parabens among pregnant women in puerto rico. Environ. Sci. Technol.47, 3439-3447 (2013).

31. Dietrich, J. W., Landgrafe, G. \& Fotiadou, E. H. TSH and thyrotropic agonists: Key actors in thyroid homeostasis. J. Thyroid Res. 2012, (2012).

32. Romero, R., Scoccia, B., Mazor, M., Wu, Y. K. \& Benveniste, R. Evidence for a local change in the progesterone/ estrogen ratio in human parturition at term. Am. J. Obstet. Gynecol.159, 657-660 (1988).

33. Pettker, C., Goldberg, J., El-Sayed, Y. \& Copel, J. Committee Opinion No 700: Methods for Estimating the Due Date. Obstet. Gynecol.129, e150-e154 (2017).

34. Villar, J. et al. International standards for newborn weight, length, and head circumference by gestational age and sex: the Newborn Cross-Sectional Study of the INTERGROWTH-21st Project. Lancet (London, England).384, 857-868 (2014).

35. Moran, D., Mcgarrigle, H. H. \& Lachelin, G. C. Lack of normal increase in saliva estriol / progesterone ratio in women with labor induced at 42 weeks' gestation.Am. J. Obstet. Gynecol.167, (1983).

36. Di Carlo, G., Giardina, I., Clerici, G., Brillo, E. \& Gerli, S. Progesterone in normal and pathological pregnancy. Horm. Mol. Biol. Clin. Investig.27, 35-48 (2016).

37. Byrns, M. C. Regulation of progesterone signaling during pregnancy: Implications for the use of progestins for the prevention of preterm birth. J. Steroid Biochem. Mol. Biol.139, 173-181 (2014).

38. Mesiano, S. \& Welsh, T. N. Steroid hormone control of myometrial contractility and parturition. Semin. Cell Dev. Biol.18, 321-331 (2007).

39. Snegovskikh, V. \& Park, J. S. Endocrinology of Parturition. Endocrinol. Metab. Clin. North Am.35, 173-191 (2006).

40. Challis, J. R. G., Matthews, S. G., Gibb, W. \& Lye, S. J. \& C, D. P. J. R. G. Endocrine and Paracrine Regulation of Birth at Term and Preterm. Endocr. Rev.21, 514-550 (2000).

41. Darne, J., Mcgarrigle, H. \& Lachelin, G. Increased saliva oestriol to progesterone ratio before idiopathic preterm delivery: a possible predictor for preterm labour? Br. Med. J.294, 270-272 (1987).

42. Johns, L. E. et al. Longitudinal profiles of thyroid hormone parameters in pregnancy and associations with preterm birth. PLoS One.12, 1-15 (2017).

43. Mancini, A. et al. Thyroid Hormones, Oxidative Stress, and Inflammation. Mediators Inflamm. 2016, 6757154 (2016). 
44. Moore, T. A., Ahmad, I. M. \& Zimmerman, M. C. Oxidative Stress and Preterm Birth: An Integrative Review. Biol. Res. Nurs.20, 497-512 (2018).

45. Cappelletti, M., Della Bella, S., Ferrazzi, E., Mavilio, D. \& Divanovic, S. Inflammation and preterm birth. J. Leukoc. Biol.99, 67-78 (2016).

46. Ferguson, K. K. et al. Environmental phthalate exposure and preterm birth in the PROTECT birth cohort. Environ. Int.132, 105099 (2019).

47. Johns, L. E., Ferguson, K. K., McElrath, T. F., Mukherjee, B. \& Meeker, J. D. Associations between Repeated Measures of Maternal Urinary Phthalate Metabolites and Thyroid Hormone Parameters during Pregnancy. Environ. Health Perspect.124, 1808-1815 (2016).

48. Cathey, A. L. et al. Associations of Phthalates and Phthalate Replacements With CRH and Other Hormones Among Pregnant Women in Puerto Rico. J. Endocr. Soc.3, 1127-1149 (2019).

49. Tache, V. et al. Population-based biomarker screening and the development of severe preeclampsia in California.American journal of obstetrics and gynecologyol. 211 (2014).

50. Medici, M. et al. Maternal early-pregnancy thyroid function is associated with subsequent hypertensive disorders of pregnancy: the generation R study. J. Clin. Endocrinol. Metab.99, E2591-8 (2014).

51. Zhang, Y. et al. Maternal low thyroxin levels are associated with adverse pregnancy outcomes in a Chinese population. PLoS One.12, e0178100 (2017).

52. Korevaar, T. I. M. et al. The Risk of Preeclampsia According to High Thyroid Function in Pregnancy Differs by hCG Concentration. J. Clin. Endocrinol. Metab.101, 5037-5043 (2016).

53. Barjaktarovic, M. et al. Human chorionic gonadotropin and risk of pre-eclampsia: prospective populationbased cohort study. Ultrasound Obstet. Gynecol.54, 477-483 (2019).

54. Lala, P. K. \& Chakraborty, C. Factors regulating trophoblast migration and invasiveness: possible derangements contributing to pre-eclampsia and fetal injury. Placenta.24, 575-587 (2003).

55. Roberts, D. J. \& Post, M. D. The placenta in pre-eclampsia and intrauterine growth restriction. J. Clin. Pathol.61, 1254-1260 (2008).

56. Boeldt, D. S. \& Bird, I. M. Vascular adaptation in pregnancy and endothelial dysfunction in preeclampsia. J. Endocrinol.232, R27-R44 (2017).

57. Morisset, A. S. et al. Androgens in the maternal and fetal circulation: association with insulin resistance. J. Matern. Fetal. Neonatal Med.26, 513-519 (2013).

58. Yang, S., Shi, F. T., Leung, P. C. K., Huang, H. F. \& Fan, J. Low Thyroid Hormone in Early Pregnancy Is Associated With an Increased Risk of Gestational Diabetes Mellitus. J. Clin. Endocrinol. Metab.101, 42374243 (2016).

59. Rawal, S. et al. A Longitudinal Study of Thyroid Markers Across Pregnancy and the Risk of Gestational Diabetes. J. Clin. Endocrinol. Metab.103, 2447-2456 (2018).

60. Ategbo, J. M. et al. Modulation of adipokines and cytokines in gestational diabetes and macrosomia. J. Clin. Endocrinol. Metab.91, 4137-4143 (2006).

61. Boelen, A., Platvoet-Ter Schiphorst, M. C. \& Wiersinga, W. M. Association between serum interleukin-6 and serum 3,5,3'-triiodothyronine in nonthyroidal illness. J. Clin. Endocrinol. Metab.77, 1695-1699 (1993).

62. Verburg, P. E. et al. Sexual Dimorphism in Adverse Pregnancy Outcomes - A Retrospective Australian Population Study 1981-2011. PLoS One.11, e0158807 (2016). 
63. Retnakaran, R. \& Shah, B. R. Fetal Sex and the Natural History of Maternal Risk of Diabetes During and After Pregnancy. J. Clin. Endocrinol. Metab.100, 2574-2580 (2015).

64. Sheiner, E. et al. Gender does matter in perinatal medicine. Fetal Diagn. Ther.19, 366-369 (2004).

65. Retnakaran, R. et al. Fetal sex and maternal risk of gestational diabetes mellitus: the impact of having a boy. Diabetes Care.38, 844-851 (2015).

66. Johns, L. E. et al. Subclinical Changes in Maternal Thyroid Function Parameters in Pregnancy and Fetal Growth. J. Clin. Endocrinol. Metab.103, 1349-1358 (2018).

67. Leon, G. et al. Maternal thyroid dysfunction during gestation, preterm delivery, and birthweight. The Infancia y Medio Ambiente Cohort, Spain. Paediatr. Perinat. Epidemiol.29, 113-122 (2015).

68. Sferruzzi-Perri, A. N., Vaughan, O. R., Forhead, A. J. \& Fowden, A. L. Hormonal and nutritional drivers of intrauterine growth. Curr. Opin. Clin. Nutr. Metab. Care.16, 298-309 (2013).

69. Forhead, A. J. \& Fowden, A. L. Thyroid hormones in fetal growth and prepartum maturation. J. Endocrinol.221, R87-R103 (2014).

\section{Figures}

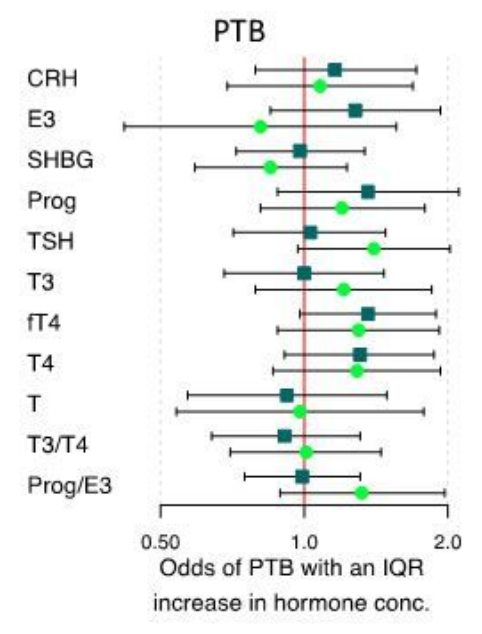

SGA

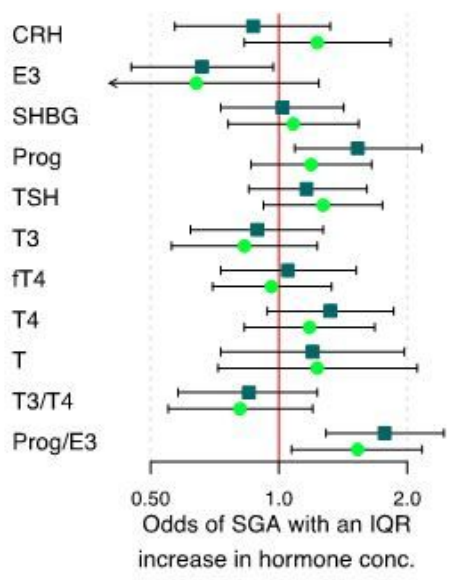

Spont. PTB

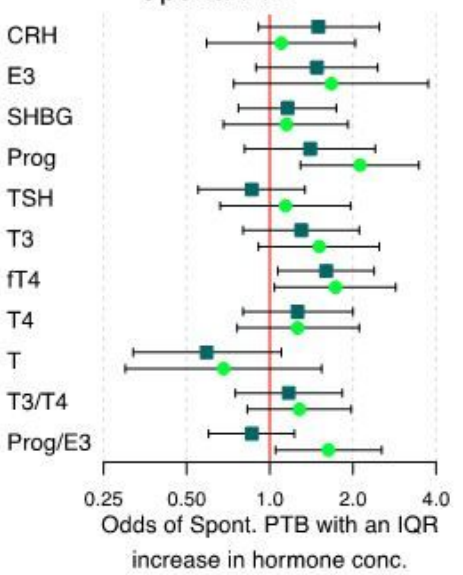

LGA

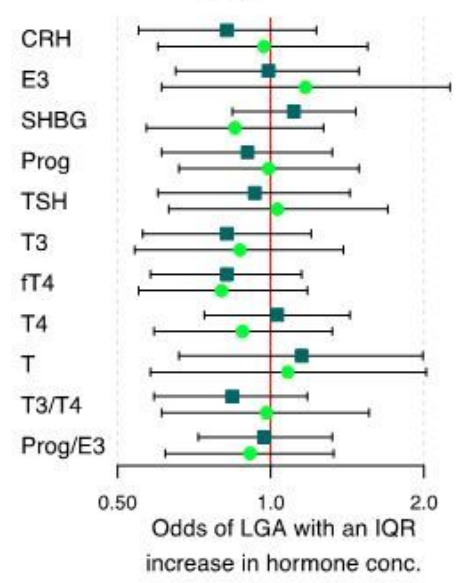

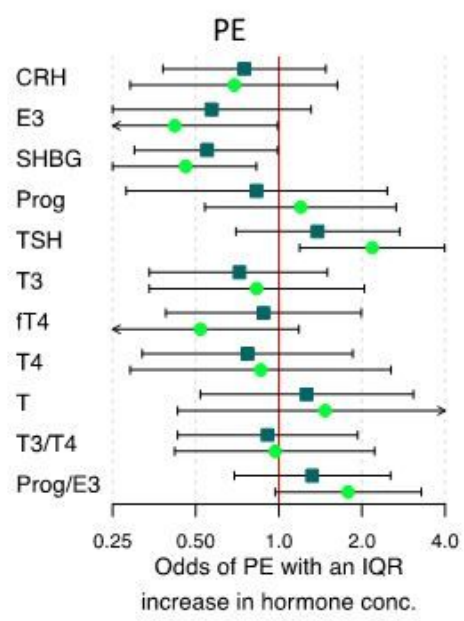

increase in hormone conc.

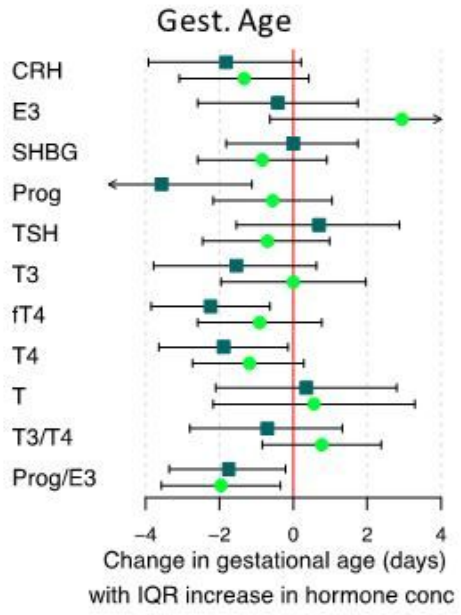

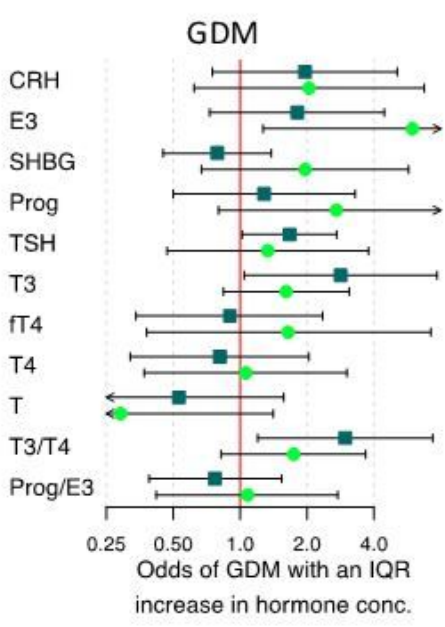

Birth Weight Z-Score

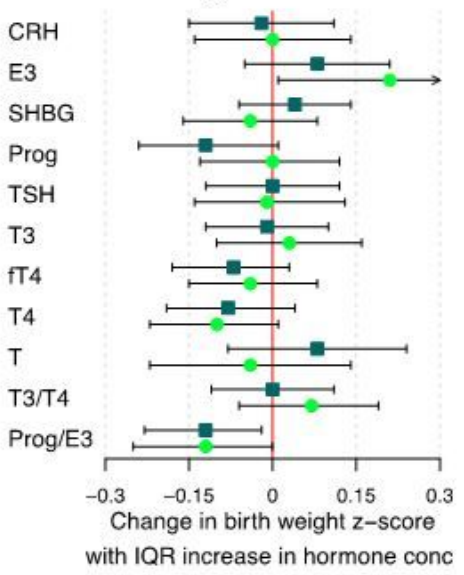

\section{Figure 1}


Differential associations between birth outcomes and hormones measured at 18 and 26 weeks' gestation. Dark green boxes represent the effect estimates for hormones measured at 18 weeks, light green circles represent the effect estimates for hormones measured at 26 weeks, and black bars represent the $95 \%$ confidence interval. The vertical red line represents the null value. IQR: Interquartile range.

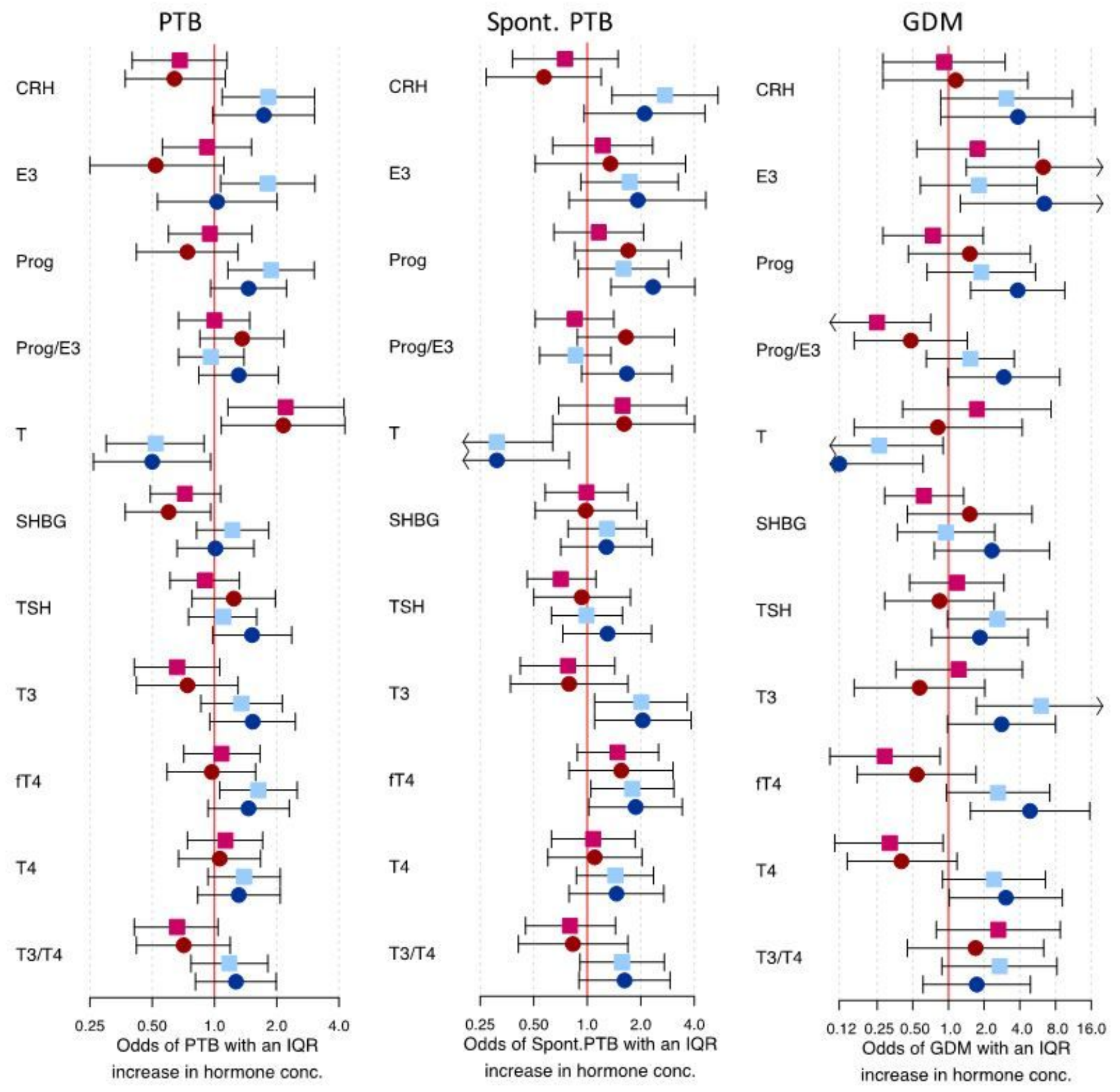

Figure 2

Differential associations between birth outcomes and hormones measured at 18 and 26 weeks based on fetal sex. Pink boxes represent the effect estimates for hormones measured at 18 weeks among female pregnancies, red circles represent the effect estimates for hormones measured at 26 weeks among female pregnancies, light blue boxes represent the effect estimates for hormones measured at 18 weeks among male pregnancies, dark 
blue circles represent the effect estimates for hormones measured at 26 weeks among male pregnancies, and black bars represent the $95 \%$ confidence interval. The vertical red line represents the null value. IQR: Interquartile range.

\section{Supplementary Files}

This is a list of supplementary files associated with this preprint. Click to download.

- SupplementaryTables.docx 